University of New Orleans

ScholarWorks@UNO

$1-4-2007$

\title{
Polarizing properties of embedded symmetric trilayer stacks under conditions of frustrated total internal reflection
}

Rasheed M.A. Azzam

University of New Orleans, razzam@uno.edu

Siva R. Perla

Follow this and additional works at: https://scholarworks.uno.edu/ee_facpubs

Part of the Electrical and Electronics Commons, and the Optics Commons

\section{Recommended Citation}

Rasheed M. A. Azzam and Siva R. Perla, "Polarizing properties of embedded symmetric trilayer stacks under conditions of frustrated total internal reflection: erratum," Appl. Opt. 46, 431-433 (2007).

This Article is brought to you for free and open access by the Department of Electrical Engineering at ScholarWorks@UNO. It has been accepted for inclusion in Electrical Engineering Faculty Publications by an authorized administrator of ScholarWorks@UNO. For more information, please contact scholarworks@uno.edu. 


\title{
Polarizing properties of embedded symmetric trilayer stacks under conditions of frustrated total internal reflection: erratum
}

\author{
Rasheed M. A. Azzam and Siva R. Perla
}

\begin{abstract}
An error in the application of the design procedure described in a previous paper [Appl. Opt. 45, 1650 (2006)] has been corrected, and new revised figures are included in this erratum. (c) 2007 Optical Society of America

OCIS codes: $\quad 310.0310,230.5440,240.0310$.
\end{abstract}

In a recent paper ${ }^{1}$ we reported on the polarizing properties of a transparent symmetric trilayer stack of refractive indices $n_{1}, n_{2}, n_{1}$ that is embedded in a transparent high-index prism of refractive index $n_{0}$. The design procedure in Section 2 of Ref. 1 and the analytical proof given in Appendix A are correct. However, an error in the application of the design procedure has led to errors in the results presented in Sections 3, 4, and 5, which have to be corrected.

The error occurred in the recovery of the normalized thickness $Z_{2}$ of the high-index center layer from the complex exponential function $X_{2}$ using Eqs. (4) and (8). Figure 1 shows the correct relationship between the normalized film thicknesses $Z_{2}$ and $Z_{1}$ for zero reflection of the $p$ polarization $\left(r_{p}=0\right)$ at angles of incidence $\phi_{0}$ from $45^{\circ}$ to $85^{\circ}$ in steps of $5^{\circ}$ for $\mathrm{MgF}_{2}\left(n_{1}=1.38\right)-\mathrm{ZnS}\left(n_{2}=2.35\right)-\mathrm{MgF}_{2}\left(n_{1}=1.38\right)$ trilayers embedded in a $\mathrm{ZnS}\left(n_{0}=2.35\right)$ substrate in the visible. Figure 1, which shows a family of nonintersecting $Z_{2}$-versus- $Z_{1}$ curves, should replace Fig. 2 of Ref. 1 . Figures 3 and 4 in Ref. 1 for the reflectance $R_{s}=\left|r_{s}\right|^{2}$ of the orthogonal $s$ polarization as a function of $Z_{1}$ remain unchanged, unaffected by the error. However, Fig. 5 of Ref. 1 has become irrelevant, since no point of intersection appears in Fig. 1.

Figure 2 shows the corresponding family of nonintersecting $Z_{2}$-versus- $Z_{1}$ curves for zero reflection of

R. Azzam (rasheed.azzam@gmail.com; razzam@uno.edu) and S. R. Perla are with Department of Electrical Engineering, University of New Orleans, New Orleans, Louisiana 70148, USA.

Received for publication 2 November 2006; posted 2 November 2006 (Doc. ID 76703); published 4 January 2007.

0003-6935/07/030431-03\$15.00/0

(C) 2007 Optical Society of America the $s$ polarization $\left(r_{s}=0\right)$ at the same angles of incidence and for the same material system as in Fig. 1. Figure 2 should replace Fig. 6 of Ref. 1. Figures 7 and 8 in Ref. 1 for the reflectance $R_{p}=$ $\left|r_{p}\right|^{2}$ of the orthogonal $p$ polarization remain unchanged, unaffected by the error. Figure 9 of Ref. 1 is now irrelevant, since no point of intersection appears in Fig. 2.

The absence of a common point of intersection in Fig. 2 renders invalid the claim of a wide-angle $r_{s}=0$ polarizer, which was presented in Section 4 (Figs. 10 and 11) of Ref. 1. Figure 3 gives the correct

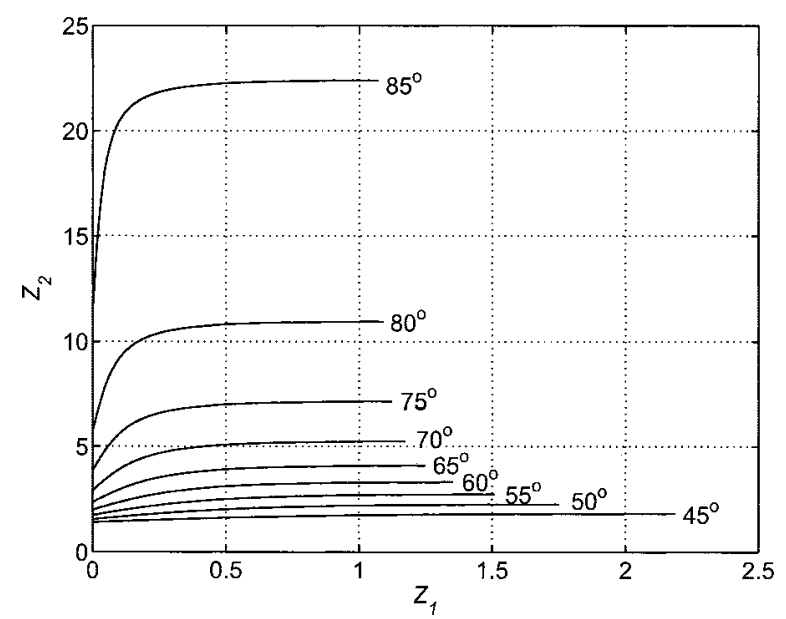

Fig. 1. Normalized layer thicknesses $Z_{2}$-versus- $Z_{1}$ such that $r_{p}=0$ at angles of incidence $\phi_{0}$ from $45^{\circ}$ to $85^{\circ}$ in steps of $5^{\circ}$, for $\mathrm{MgF}_{2}-\mathrm{ZnS}-\mathrm{MgF}_{2}$ trilayers embedded in a $\mathrm{ZnS}$ substrate with refractive indices $n_{0}=2.35(\mathrm{ZnS}), n_{1}=1.38\left(\mathrm{MgF}_{2}\right)$, and $n_{2}=$ $2.35(\mathrm{ZnS})$ in the visible. This figure should replace Fig. 2 of Ref. 1. 


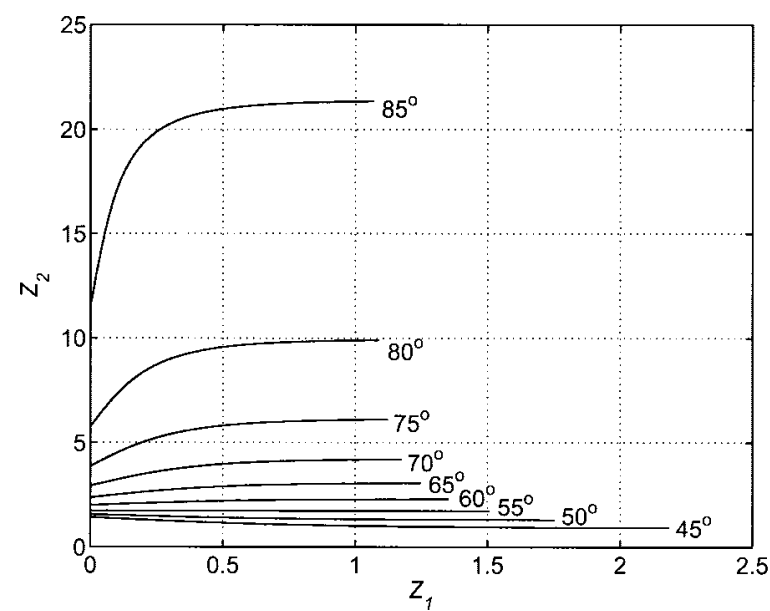

Fig. 2. Normalized layer thicknesses $Z_{2}$-versus- $Z_{1}$ such that $r_{s}$ $=0$ at angles of incidence $\phi_{0}$ from $45^{\circ}$ to $85^{\circ}$ in steps of $5^{\circ}$, for $\mathrm{MgF}_{2}-\mathrm{ZnS}-\mathrm{MgF}_{2}$ trilayers embedded in a $\mathrm{ZnS}$ substrate with refractive indices $n_{0}=2.35(\mathrm{ZnS}), n_{1}=1.38\left(\mathrm{MgF}_{2}\right)$, and $n_{2}=$ $2.35(\mathrm{ZnS})$ in the visible. This figure should replace Fig. 6 of Ref. 1.

angular response of the same trilayer system as specified in the caption of Fig. 10 of Ref. 1. This particular trilayer achieves $r_{s}=0$ and $R_{p}=\left|r_{p}\right|^{2}=0.9756$ at $\phi_{0}=55^{\circ}$ angle of incidence (as before), and functions as an orthogonal polarizer, $r_{p}=0, R_{s}=\left|r_{s}\right|^{2}=$ 0.7258 at another angle $\phi_{0}=45.78^{\circ}$. The spectral response of this system in the $600-700 \mathrm{~nm}$ range (which would replace Fig. 12 of Ref. 1) is not good enough to warrant inclusion here.

Figure 4 shows the correct family of $Z_{2}$-versus- $Z_{1}$ curves for zero reflection of the $p$ polarization $\left(r_{p}\right.$ $=0$ ) at angles of incidence $\phi_{0}$ from $45^{\circ}$ to $85^{\circ}$ in steps of $5^{\circ}$ for $\mathrm{CaF}_{2}\left(n_{1}=1.4\right)-\mathrm{Ge}\left(n_{2}=4.0\right)-\mathrm{CaF}_{2}\left(n_{1}=\right.$ 1.4) trilayers embedded in a $\mathrm{ZnS}\left(n_{0}=2.2\right)$ substrate in the IR. Figure 4 should replace Fig. 13 of Ref. 1. Figure 14 in Ref. 1 for the reflectance $R_{s}=\left|r_{s}\right|^{2}$ of the

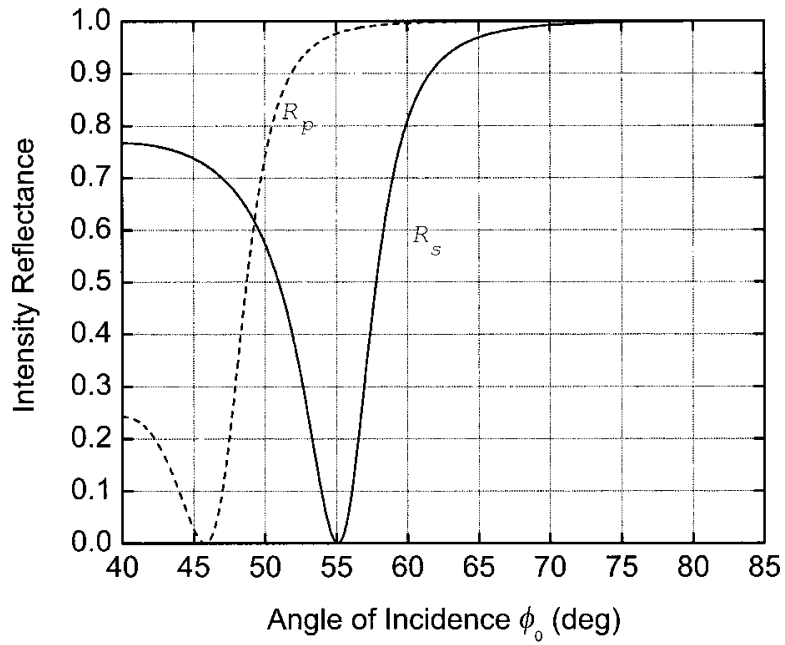

Fig. 3. Angular reflectance response for $p$ - and $s$-polarized light (at wavelength $\lambda=633 \mathrm{~nm}$ ) of the same embedded trilayer system as specified in the caption of Fig. 10 of Ref. 1.

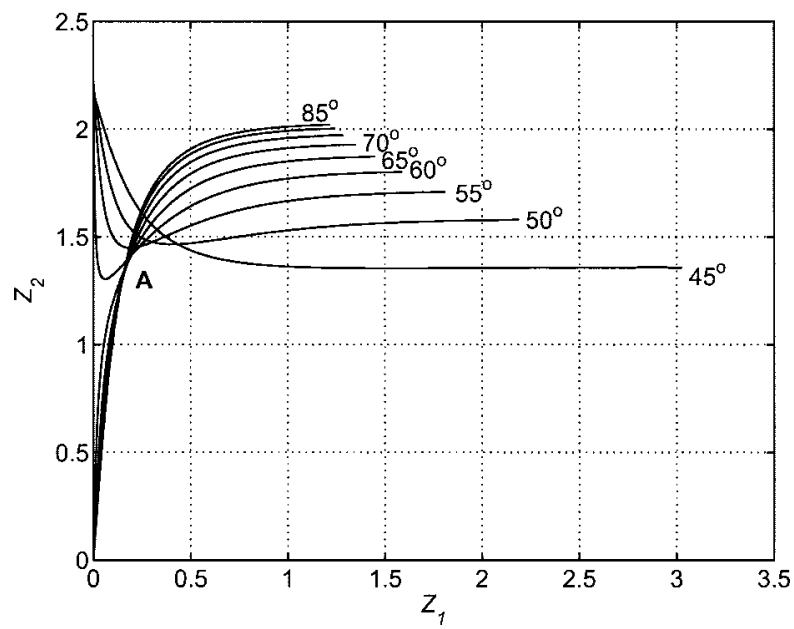

Fig. 4. Family of $Z_{2}$-versus- $Z_{1}$ curves for zero reflection of the $p$ polarization $\left(r_{p}=0\right)$ at incidence angles $\phi_{0}$ from $45^{\circ}$ to $85^{\circ}$ in steps of $5^{\circ}$ for $\mathrm{CaF}_{2}\left(n_{1}=1.4\right)-\mathrm{Ge}\left(n_{2}=4.0\right)-\mathrm{CaF}_{2}\left(n_{1}=1.4\right)$ trilayers embedded in a $\mathrm{ZnS}\left(n_{0}=2.2\right)$ substrate in the $1 \mathrm{R}$. This figure should replace Fig. 13 of Ref. 1.

$s$ polarization as a function of $Z_{1}$ remains unchanged, unaffected by the error.

In Fig. 4, a common point of intersection A appears for $Z_{2}$-versus- $Z_{1}$ curves that correspond to incidence angles $\phi_{0} \geq 60^{\circ}$. The $Z_{2}$-versus- $Z_{1}$ curves at the two angles $\phi_{0}=60^{\circ}$ and $75^{\circ}$ intersect at $\left(Z_{1}=\right.$ $\left.0.177801, Z_{2}=1.396668\right)$. The angular response of the design that corresponds to this point of intersection, at wavelength $\lambda=10.6 \mu \mathrm{m}$, is shown in Fig. 5. It is apparent that the trilayer functions as an effective antireflection coating for the $p$ polarization over a wide range of angles at oblique incidence. However, the associated $s$ reflectance is not high enough to qualify the system as an effective polarizing beam splitter, but may be adequate for the device to operate as a wide-angle reflection polarizer only.

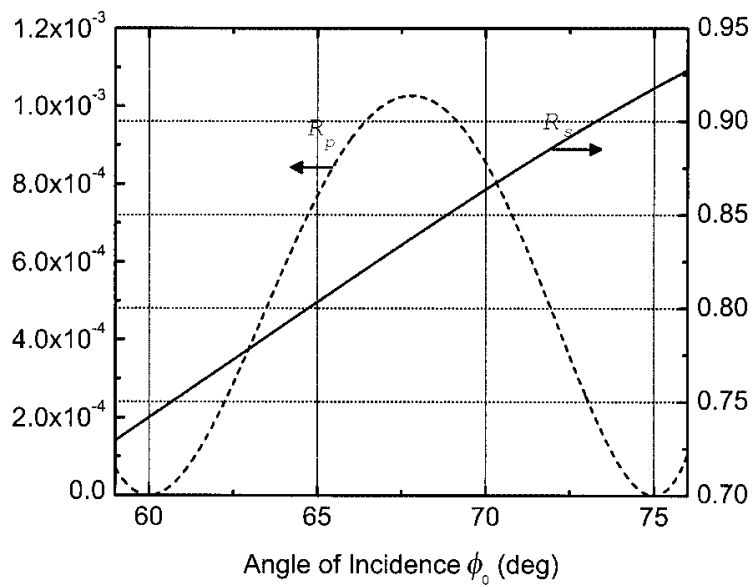

Fig. 5. Angular reflectance response for $p$ - and $s$-polarized light (at wavelength $\lambda=10.6 \mu \mathrm{m}$ ) of an embedded trilayer design that corresponds to the point of intersection $A\left(Z_{1}=0.177801, Z_{2}=\right.$ 1.396668) of the two curves in Fig. 4 that correspond to $\phi_{0}=60^{\circ}$ and $75^{\circ}$. 


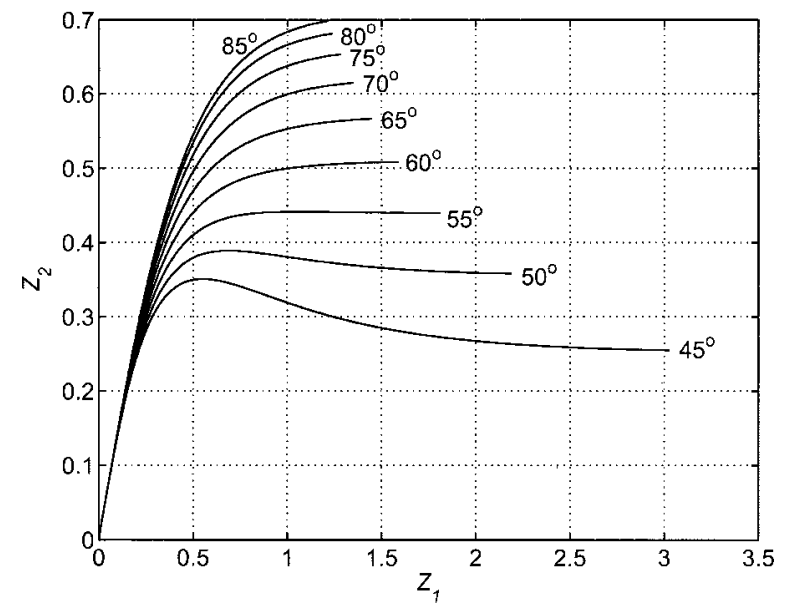

Fig. 6. Family of $Z_{2}$-versus- $Z_{1}$ curves for zero reflection of the $s$ polarization $\left(r_{s}=0\right)$ at incidence angles $\phi_{0}$ from $45^{\circ}$ to $85^{\circ}$ in steps of $5^{\circ}$ for $\mathrm{CaF}_{2}\left(n_{1}=1.4\right)-\mathrm{Ge}\left(n_{2}=4.0\right)-\mathrm{CaF}_{2}\left(n_{1}=1.4\right)$ trilayers embedded in a $\mathrm{ZnS}\left(n_{0}=2.2\right)$ substrate in the IR. This figure should replace Fig. 15 of Ref. 1.
Figure 6 shows the correct family of $Z_{2}$-versus- $Z_{1}$ curves for zero reflection of the $s$ polarization at angles of incidence $\phi_{0}$ from $45^{\circ}$ to $85^{\circ}$ in steps of $5^{\circ}$ for $\mathrm{CaF}_{2}\left(n_{1}=1.4\right)-\mathrm{Ge}\left(n_{2}=4.0\right)-\mathrm{CaF}_{2}\left(n_{1}=1.4\right)$ trilayers embedded in a $\mathrm{ZnS}\left(n_{0}=2.2\right)$ substrate in the IR. Figure 6 replaces Fig. 15 of Ref. 1. Figure 16 in Ref. 1 for the reflectance $R_{p}=\left|r_{p}\right|^{2}$ of the $p$ polarization as a function of $Z_{1}$ is unchanged, unaffected by the error.

In summary, an error in the application of the design procedure described in Ref. 1 has been identified, and the corrected versions of five figures in Ref. 1 are included in this erratum.

We thank J. A. Dobrowolski for providing an independent verification of the correct angular response of an embedded trilayer shown in Fig. 3.

\section{Reference}

1. R. M. A. Azzam and S. R. Perla, "Polarizing properties of embedded symmetric trilayer stacks under conditions of frustrated total internal reflection,” Appl. Opt. 45, 1650-1656 (2006). 